1 UNC Gillings School of Global Public Health, North Carolina, USA

2 San Francisco, California, USA

3 Boston University, Boston, Massachusetts, USA

4 Division of Health Policy and Management, Department of Public Health Sciences, University of California - Davis, Davis, California, USA

Cite this as: BMJ 2022;376:0344 http://dx.doi.org/10.1136/bmj.0344 Published: 8 February 2022

\section{Putting equity at the heart of the US government's rollout of covid tests and masks}

\section{Federal government must improve the infrastructure that supports people's access to masks and tests, say Deshira D Wallace and colleagues}

\author{
Deshira D Wallace, ${ }^{1}$ Kristin Urquiza, ${ }^{2}$ Julia Raifman, ${ }^{3}$ Michelle Ko ${ }^{4}$
}

The Omicron variant of covid-19 continues to surge throughout the US, and Americans are stuck repeating the same self-destructive cycles again: political leaders debate mitigation policies, while hundreds of thousands of people become ill and health systems are overwhelmed. In the wake of the Omicron variant's arrival, the US Centers for Disease Control and Prevention released revised guidelines for isolation, quarantine, testing, and upgrading mask quality. Still, Americans are asked to engage in individualised actions, regardless of their access to resources to protect themselves and their communities. This is the antithesis of a public health response. What we need is an approach based on equity that improves access to masks and tests for those with the least access.

On 18 January 2022, the US government launched a website where Americans could order one set of up to four rapid antigen tests per household, ${ }^{1}$ to be delivered for free by the US Postal Service. ${ }^{2}$ Within the week, the White House announced that they would release 400 million free $\mathrm{N} 95$ masks from the federal stockpile for public use, ${ }^{3}$ to be distributed through pharmacies and community health centres.

These programmes have come nearly a year after President Biden entered office and put forward a plan to use the Defence Production Act to scale up the provision of rapid tests and high quality masks, a promise that was never fulfilled. ${ }^{4}$ While the recent releases of tests and masks are steps in the right direction, they are late and insufficient.

As Omicron spreads across the US, people on low incomes continue to have the most exposure to the virus and the least access to vaccines, boosters, high quality masks, or rapid tests to protect themselves. Across the US, many people, including those who are older, living with disabilities, or with young children, have waited hours to get PCR tests (both free and paid for) at testing sites. Many were covid positive.

These federal programmes are a welcome response to these shortages, but still fail to centre those who are most vulnerable. ${ }^{5}$ The distributions already came too late in the Omicron surge for those in immediate need of tests due to a high risk of exposure, such as individuals who have essential jobs. ${ }^{6}$ For those requesting free tests, the number is too few: four tests per household does not fit the reality of how multigenerational households, families with greater than four people, and roommates live. Ordering from the website requires individuals having reliable internet access, digital devices, and literacy in English, Spanish, or simplified Chinese, although ${ }^{2}$ an option to order by phone overcomes some hurdles for English speakers.

For those needing more at home tests, the government has directed Americans to use their private health insurance. For many people on a reimbursement model, this will mean they'll need to have sufficient money upfront, before navigating a complicated reimbursement process, without a guarantee of getting all their money back. ${ }^{7}$ For others, their coverage may allow them to purchase a test directly or pick one up for free from a network pharmacy, ${ }^{7}$ provided they can navigate these options. Medicaid also covers at home tests, but the rules vary by state, including how many and whether or not individuals must first obtain a prescription. Medicare will not offer tests until later this spring. ${ }^{8}$ In short, policies based upon health insurance systems favour those privileged with time, money, and internet savvy, laying the burden on those who need the most protection.

The new federal mask programme also reflects a lack of preparation. We do not have enough masks to adequately protect our population. The administration has signalled they will prioritise mask distribution to the communities that are most affected, with the use of pharmacies and community health centres a good start. However, long standing inequities in neighbourhood investment have led to pharmacy deserts in Black and Hispanic/Latinx neighbourhoods. ${ }^{9}$ Similarly, community health centres have been critical partners throughout the pandemic, but due to the limited number of them, they care for only one in five Medicaid beneficiaries. ${ }^{10}$ Despite the government's plans to widely distribute high quality masks, the communities most in need may not be able to access them.

Lastly, the one time nature of the mask distribution, just like the tests, assumes a time limit to the pandemic, when more than once new variants and rising case numbers have caught us unprepared. One time distributions run counter to the necessity of ensuring the continuous availability and accessibility of public health interventions in all communities, for use in current and future covid surges.

The federal response should make the following changes to improve access and reduce inequities in covid-19 outcomes:

Mail free masks and rapid tests to every household in the country based on the number of occupants and continue to do so at the start of every surge in case numbers. 
Distribute free masks and at home rapid or PCR tests in public spaces throughout our communities-from libraries to grocery stores.

\$scale up the production of masks and tests and reduce the price to improve accessibility throughout the pandemic.

Develop clearly communicated federal policies (which are driven by data ${ }^{11}$ ) on when and where to wear masks, the types of masks to wear, and, similarly, when to use at home tests.

Ultimately, the costs of having an insufficient supply of high quality masks and tests far exceed the cost of providing these supplies to people in low income populations who remain most exposed to the virus. With the Omicron surge, the cost was measured in hours waiting in the cold to be tested, leaving schools and businesses with no choice but to close when many people became infected, in preventable hospital admissions, overwhelmed hospitals, and deaths.

When we put the onus on individuals to take action to prevent covid infections and transmission, without providing institutional support, we force people into the trap of blaming each other for a monumental collective problem. To facilitate people making the "right" choices, protecting themselves and each other, our federal government must improve the public health infrastructure that supports people's access to masks and tests. ${ }^{12}$

Acknowledgements: We want to acknowledge Marked By COVID co-founder Christine Keeves, MPH, and the Marked By COVID community for their support and perspectives.

Competing interests: none declared.

Provenance and peer review: Commissioned; not externally peer reviewed.

1 Bogage J. Biden plan to ship 500 million coronavirus test kits transforms Postal Service into relief agency. The Washington Post. 21 January 2022. https://www.washingtonpost.com/business/2022/01/21/usps-coronavirus-test-kits/

2 Place your order for free at-home COVID-19 tests. U.S. Postal Service. Updated January 20, 2022. https://special.usps.com/testkits

3 Treisman R. The Biden administration will give out 400 million free N95 masks. NPR. 19 January 192022. https://www.npr.org/2022/01/19/1074037421/the-biden-administration-will-give-out400-million-free-n95-masks

4 President Joseph Biden Jr. National strategy for the COVID-19 response and pandemic preparedness. The White House. 21 January 2021. https://www.whitehouse.gov/wp-content/uploads/2021/01/National-Strategy-for-the-COVID-19-Response-and-Pandemic-Preparedness.pdf

5 Fox J. The current COVID-19 surge, eugenics, and health-based discrimination. The Petrie-Flom Center, Harvard Law. 23 July 2021. https://blog.petrieflom.law.harvard.edu/2021/07/23/covideugenics-health-based-discrimination/

6 Wolfe R, Harknett K, Schneider D. Inequalities at Work and The Toll Of COVID-19. Health Affairs Health Policy Brief 2021;4. doi: 10.1377/hpb20210428.863621.

7 Dawson L, Amin K, Kates J, Cox C. How are private insurers covering at-home rapid COVID tests? Kaiser Family Foundation. 20 January 2022. https://www.kff.org/policy-watch/how-are-privateinsurers-covering-at-home-rapid-covid-tests/

8 Rudowitz R. Medicaid and At-home COVID-19 Tests. Kaiser Family Foundation. 20 January 2022. https://www.kff.org/policy-watch/medicaid-and-at-home-covid-19-tests/

9 Guadamuz JS, Wilder JR, Mouslim MC, Zenk SN, Alexander GC, Qato DM. Fewer Pharmacies In Black And Hispanic/Latino Neighborhoods Compared With White Or Diverse Neighborhoods, 2007-15. Health Aff (Millwood) 2021;40:802-11. doi: 10.1377/hlthaff.2020.01699. pmid: 33939507

10 NACHC. Health Centers Finding COVID. National Association of Community Health Centers. 6 December 2021. https:/www.nachc.org/coronavirus/health-centers-fighting-covid-19/

11 Raifman J, Skinner A. State policies can provide clear guidance on when to put on and take off masks - with benefits to health, education, and the economy. The Conversation. 10 August 2021. https://theconversation.com/state-policies-can-provide-clear-guidance-on-when-to-put-on-andtake-off-masks-with-benefits-to-health-education-and-the-economy-165521

12 Green T, Venkataramani AS. Trade-offs and policy options - using insights from economics to inform public health policy. N Engl J Med 2022;386:405-8. doi: 10.1056/NEJMp2104360. pmid: 35089666 